TITLE:

\title{
Satellite Links with Brunnian Properties
}

$\operatorname{AUTHOR}(S)$ :

Kanenobu, Taizo

CITATION:

Kanenobu, Taizo. Satellite Links with Brunnian Properties. 数理解析研 究所講究録 1985, 542: 27-36

ISSUE DATE:

1985-01

URL:

http://hdl.handle.net/2433/98775

RIGHT: 
Satellite Links with Brunnian Properties

\section{Taizo Kanenobu (金信泰造)}

We shall work throughout in the PL category. An n-link with $\mathrm{m}$ components is a locally flat oriented submanifold of the oriented $(n+2)$-sphere $s^{n+2}$ homeomorphic to $m$ disjoint copies of $\mathrm{s}^{\mathrm{n}}$. An $\mathrm{n}$-knot is an $\mathrm{n}$-link with one component. A trivial $\mathrm{n}$-link is one whose components bound disjoint locally flat $(n+1)$-disks $B^{n+1}$ in $S^{n+2}$. An $n-l i n k \quad L$ is splittable if. there exists an $(n+2)$-disk $B^{n+2}$ in $S^{n+2}$ satisfying $L \cap B^{n+2}$ $\neq \varnothing, \quad L \cap \partial B^{n+2}=\varnothing$, and $L \cap\left(S^{n+2}-B^{n+2}\right) \neq \varnothing$, where $\partial B^{n+2}$ is the boundary of $B^{n+2}$.

Let $o$ be the family of those subsets $S$ of $I=\{1,2$, $\ldots, \mathrm{m}\}$ for which the sublink $L_{S}=\bigcup_{i \in S} L_{i}$ of an $n-l i n k \quad L=L_{1} U$ $\mathrm{L}_{2} \cup \ldots \cup \mathrm{L}_{\mathrm{m}}$ does not split. Then we call $\mathrm{L}$ has the Brunnian property of type $\theta$. For the convenience we assume that $\phi,\{i\}$ $\notin$ or for all $i \in I$. In this family of subsets $\alpha$, the following condition must be satisfied:

$$
\text { (*) If } S, T \in Q \text { and } S \cap T \neq \varnothing \text {, then } S \cup T \in Q \text {. }
$$

Conversely we prove: 
Theorem. Suppose $\mathrm{n} \geqq 1$ and $\mathrm{m} \geqq 2$. Let or be a family of subsets of I satisfying the condition (*). Then there exists an $n$-link with $m$ components with the Brunnian property of type $\boldsymbol{\theta}$.

This theorem is previously obtained by H. Debrunner [3] for $\mathrm{n} \geqq 2$, using a ribbon $\mathrm{n}$-link. Our example is a satellite link, which is defined in a similar way that a satellite knot is defined in [11, pp.110-113] and [7]. As partial results, the following are known: Let $f_{k}, 2 \leqq k \leqq m$, be the family of all the subsets of I consisting of $k$ or more elements. An $n-l i n k$ with the Brunnian property of type $f_{k}$ is one such that no sublink with $\mathrm{k}$ or more components is splittable but every sublink with less than $k$ components is completely splittable. For $\mathrm{n}=1$ and $\mathrm{k}=\mathrm{m}$, such links were given by $\mathrm{H}$. Brunn [1], see also [11, pp.67-69]; for $n=1$ and $k \leqq m$, by $H$. Debrunner [2]. R. H. Fox [3, problem 38] asked whether examples existed for $\mathrm{n}=2$ and $\mathrm{k} \geqq \mathrm{m}$, and T. Yanagawa [13] answered by constructing such examples using ribbon 2 -link. For $n \geqq 1$ and $\mathrm{k}=\mathrm{m}$, see also [11, pp.197-199].

A group $G$ is indecomposable (relative to free product) if $G=A * B$ implies $A=1$ or $B=1$. To prove that a link is unsplittable, we use the following fact, cf. [10, Theorem 27.1]:

Proposition. An $n$-link $L$ is nonsplittable if its group $\pi_{1}\left(S^{n+2}-L\right)$ is indecomposable. If $n=1$, then the converse is valid. 
Hence any 1-knot group is indecomposable. Moreover an n-knot group with a nontrivial center ([5]) is indecomposable $([9, \mathrm{p} .195])$.

Proof of Theorem. Let $0=\mathrm{O}_{1} \cup \mathrm{O}_{2} \cup \ldots \cup \mathrm{O}_{\mathrm{m}}$ be a trivial $\mathrm{n}$-link with $\mathrm{m}$ components. Let $\mathrm{x}_{i} \in \pi_{1}\left(\mathrm{~S}^{\mathrm{n}+2}-0\right)$ be a meridian of $O_{i}$. Let $S=\left\{i_{1}, i_{2}, \ldots, i_{k}\right\} \quad I, \quad 1 \leqq i_{1}<i_{2}<$ $\ldots<i_{k} \leqq m$. We write $F_{S}$ for the free group with basis $\left\{x_{i_{1}}\right.$, $\left.x_{i_{2}}, \ldots, x_{i_{k}}\right\}$; thus $\pi_{1}\left(s^{n+2}-o_{S}\right)=F_{S}$. Let $\alpha_{S}=\left[x_{i_{1}}, x_{i_{2}}\right.$, $\left.\ldots, x_{i_{k}}\right]$, where $\left[x_{i_{1}}, x_{i_{2}}\right]=x_{i_{1}}^{-1} x_{i_{2}}^{-1} x_{i_{1}} x_{i_{2}}$ and $\left[x_{i_{1}}, \ldots\right.$, $\left.x_{i_{j-1}}, x_{i_{j}}\right]=\left[\left[x_{i_{1}}, \ldots, x_{i_{j-1}}\right], x_{i_{j}}\right]$.

Let $\quad \alpha=\left\{s_{1}, s_{2}, \ldots, s_{r}\right\}$ and let $\alpha_{i}=\alpha_{s_{i}}$. If $n=1$, then $\alpha_{i}$ can be represented by mutually disjoint simple closed curves $l_{i}$ in $S^{3}-N(O)$, where $N(O)$ is a tubular neighborhood of 0 in $S^{3}$, such that the $\left(\# S_{i}+1\right)$-component link $\mathrm{O}_{S_{i}} \cup \ell_{i}$ has the Brunnian property of type $\mathscr{D O F}_{\# S_{i}+1}$ and that the $r$-component link $\ell_{1} \cup \ell_{2} \cup \ldots \cup \ell_{r}$ is trivial. We can always find such $\ell_{i}$ as illustrated in the figure, which consists of four circles $\mathrm{O}_{1} \cup \mathrm{O}_{2} \cup \mathrm{O}_{3} \cup \mathrm{O}_{4}$ and three curves $\ell_{1}$ $\cup \ell_{2} \cup \ell_{3}$, where $\ell_{1}, \ell_{2}$ and $\ell_{3}$ represent $\left[x_{1}, x_{2}, x_{3}\right]$, $\left[x_{3}, x_{4}\right]$ and $\left[x_{2}, x_{4}\right]$, respectively, see $[7, p .67]$. If $n \geqq 2$, we can also find mutually disjoint simple closed curves $\ell_{i}$ in $s^{n+2}-N(0)$; each $\alpha_{i}$ is represented by a unique isotopy class of $\ell_{i}([6$, Corollary 8.1 .2 and Theorem 10.1]). 
Hence in any case, if let $v_{i}$ be disjoint tubular neighborhoods of the $l_{i}$ in $s^{n+2}-N(0)$, then $s^{n+2}-$ int $v_{i}$ is homeomorphic to $S^{n} \times D^{2}$, where int $v_{i}$ is the interior of $v_{i}$. Let $K$ be an $n$-knot such that $\pi_{1}\left(s^{n+2}-K\right)$ is not infinite cyclic and indecomposable. Let $\mathrm{h}_{1}: \mathrm{s}^{\mathrm{n}+2}-$ int $\mathrm{v}_{i} \rightarrow$ $N(K)$ be a homeomorphism. Then $s^{n+2}-$ int $h_{1}\left(V_{i}\right)$ is homeomorphic to $s^{n} \times D^{2}, 2 \leqq i \leqq r$. In the same way, we inductively define homeomorphisms $h_{j}: s^{n+2}-$ int $v_{j}^{j-1} \rightarrow N(K)$, $1 \leqq j \leqq r$, where $v_{i}^{0}=v_{i}$ and $v_{i}^{j}=h\left(v_{i}^{j-1}\right), j+1 \leqq i \leqq r$. Let $\ell_{i}^{0}=\ell_{i}$ and $\ell_{i}^{j}=h_{j}\left(\ell_{i}^{j-1}\right)$. Let $L_{0}=0$ and $L^{j}=h_{j}\left(L^{j-1}\right)$, where $L_{i}^{0}=O_{i}$ and $L_{i}^{j}=h_{j}\left(L_{i}^{j-1}\right)$. We show that the iterated satellite link $L=L^{r} \quad\left(L_{i}=L_{i}^{r}\right)$, has the Brunnian property of type $a$. If $S_{i} \not \subset T<I$, then $l_{i}$ and $O_{T}$ split and if $S_{i}$ $S_{j}=\varnothing$, then $l_{i}$ and $O_{S_{j}}$ split. Thus, if $T \notin \theta$, then $L_{T}$ is splittable. Moreover, to show the contrary, we have only to prove that $L$ is nonsplittable assuming $I \in O$.

Let $S_{1}=I$ and $m>\# S_{2} \geqq \ldots \geqq \# S_{r}$. Applying the van Kampen theorem, we have the diagrams of inclusion homomorphisms:

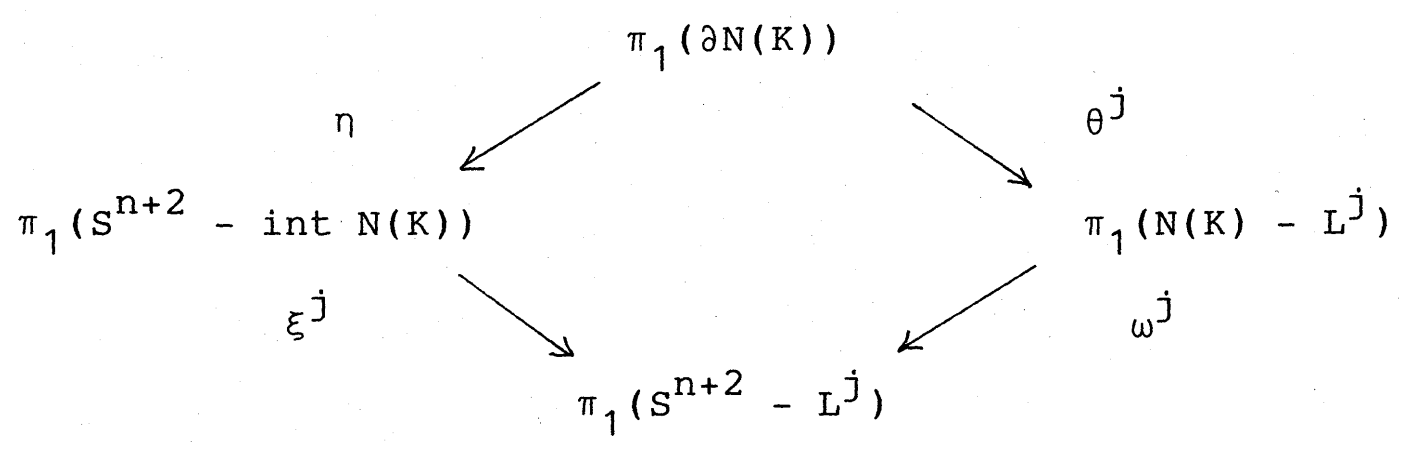


for $1 \leqq j \leqq r$. Note that $\pi_{1}\left(S^{n+2}-\right.$ int $\left.N(K)\right) \cong \pi_{1}\left(S^{n+2}-K\right)$. Since $O_{S_{j}}$ and $l_{i}$ split for $1 \leqq i \leqq j, L_{S_{j+1}}^{j}=O_{S_{j+1}}$, and so $\pi_{1}\left(S^{n+2}-L_{S_{j+1}}^{j}\right)=F_{S_{j+1}}$. By deleting the components which are not contained in $S_{j+1}$, we have an epimorphism $\psi^{j}: \pi_{1}\left(S^{n+2}\right.$ $\left.-L^{j}\right) \rightarrow F_{S} \cdot$

If both $\eta$ and $\theta^{j}$ are injective, then both $\xi^{j}$ and $\omega^{j}$ are also injective $([9, \operatorname{Sec} .4 .2])$, that is $\pi_{1}\left(S^{n+2}-L^{j}\right)$ is the free product of $\pi_{1}\left(S^{n+2}-K\right)$ and $\pi_{1}\left(N(K)-L^{j}\right)$ with an amalgamated subgroup $\pi_{1}(\partial N(K))[9$, p.207]. Further suppose that both $\pi_{1}\left(S^{n+2}-K\right)$ and $\pi_{1}\left(N(K)-L^{j}\right)$ are indecomposable, then $\pi_{1}\left(S^{n+2}-L^{j}\right)$ is indecomposable $[9, p .246]$.

Case 1. $\mathrm{n}=1$. Let $\pi_{1}(\partial N(\mathrm{~K}))=\langle\mu, \lambda \mid[\mu, \lambda]=1\rangle$, where $\mu$ is a meridian and $\lambda$ is a longitude. Since $K$ is knotted, $\eta$ is injective ([11, Theorem 4B2]). Let $f^{j}: \pi_{1}(N(K)$ $\left.-L^{j}\right) \rightarrow \pi_{1}\left(S^{3}-L^{j-1} \ell_{j}^{j-1}\right)$ be an isomorphism and $\zeta^{j-1}$ : $\pi_{1}\left(S^{3}-L^{j-1} \quad \ell_{j}^{j-1}\right) \rightarrow \pi_{1}\left(S^{3}-L^{j-1}\right)$ be an inclusion homomorphism. Then $\psi^{j-1} \zeta^{j-1} f^{j} \theta^{j}(\mu)=\alpha_{j^{\prime}}$ which has infinite order in $F_{S_{j}}\left(\left[9\right.\right.$, Sec. 1.4]). Furthermore $f^{j} \theta^{j}(\lambda)$ is a meridian of $\ell_{j}^{j+1}$, and so $\theta^{j}$ is injective. Thus $\xi^{j}$ and $\omega^{j}$ are injective.

Since any proper sublink of $O \cup \ell_{1}$ is trivial and $\ell_{1}$ represents a nontrivial element $\alpha_{1}$ in $F_{I}, O \cup \ell_{1}$ is nonsplittable, and so $\pi_{1}\left(N(K)-L^{j}\right) \cong \pi_{1}\left(S^{3}-O \ell_{\ell_{1}}\right)$ is indecomposable. In the same way, $L_{S_{j}}^{j-1} \cup \ell_{j}^{j-1}=O_{S_{j}} \cup \ell_{j}$ is 
nonsplittable. Suppose that $L^{j-1}$ is nonsplittable. Then $L^{j-1}$ $\cup e_{j}^{j-1}=\left(L_{S_{j}}^{j-1} \cup e_{j}^{j-1}\right) \cup L^{j-1}$ is also nonsplittable, and so $\pi_{1}\left(N(K)-L^{j}\right) \cong \pi_{1}\left(S^{3}-L^{j-1} \cup e_{j}^{j-1}\right)$ is indecomposable. Hence by induction on $j, \pi_{1}\left(S^{3}-L\right)$ is indecomposable.

Case 2. $n \geqq 2$. Let $\pi_{1}(\partial N(K))=\langle\mu|>$. Then $\eta(\mu)$ is a meridian of $N(K)$, and so $\eta$ is injective. Since the inclusion homomorphism $\pi_{1}\left(\left(S^{n+2}-\right.\right.$ int $\left.\left.V_{j}^{j-1}\right)-L^{j}\right) \rightarrow \pi_{1}\left(S^{n+2}-L^{j}\right)$ is isomorphic, we have an isomorphism $g^{j}: \pi_{1}\left(N(K)-L^{j}\right) \rightarrow$ $\pi_{1}\left(S^{n+2}-L^{j}\right)$ and $\psi^{j} g^{j} \theta^{j}(\mu)=\alpha_{j+1}$, which has infinite order in $F_{S_{j+1}}$, and so $\theta^{j}$ is injective. Thus $\xi^{j}$ and $\omega^{j}$ are injective.

If $\pi_{1}\left(N(K)-L^{1}\right) \cong \pi_{1}\left(S^{n+2}-L^{1}\right)$ is indecomposable, then by induction on $j, \pi_{1}\left(S^{n+2}-L\right)$ is indecomposable. Hence the proof is reduced to the lemma below.

Sublemma. Let $H_{m}=\left\langle x_{1}, x_{2}, \ldots, x_{m} \mid\left[x_{1}, x_{2}, \ldots, x_{m}\right]=1\right\rangle$. If $m \geqq 2$, then $H_{m}$ is indecomposable.

Proof. We prove by induction on $\mathrm{m} \cdot \mathrm{H}_{2}$ is free abelian of rank 2, and is indecomposable. Assume that $H_{m-1}$ is indecomposable. Let $H_{m}=A * B$. Since $\beta=\left[x_{1}, x_{2}, \ldots, x_{m-1}\right]$ and $x_{m}$ commute, either both $\beta$ and $x_{m}$ are in a conjugate of $A$ or $B$, or $B$ and $x_{m}$ are both powers of the same element [9, Corollary 4.1.6]. Considering the exponent sums on generators, the latter case cannot occur. Thus by an inner 
automorphism of $\mathrm{H}_{\mathrm{m}}$, we may suppose that $\beta \in A$ and $\mathrm{x}_{\mathrm{m}} \in$ A. Let $\mathrm{N}$ be the normal subgroup generated by $\beta$ and $\mathrm{x}_{\mathrm{m}}$ in A. Then we have $H_{m-1} \cong A / N * B$, cf. [8, Problem 4.1.5]. By inductive hypothesis, we obtain $A / N=1$ or $B=1$. If $A / N=$ 1, then $\mathrm{H}_{\mathrm{m}} \cong \mathrm{A} * \mathrm{H}_{\mathrm{m}-1}$, and so the rank (i.e., minimum number of generators) of $\mathrm{A}$ is one [8, p.192], a contradiction. This completes the proof.

Lemma. If $\mathrm{n} \geqq 2$, then $\mathrm{G}=\pi_{1}\left(\mathrm{~S}^{\mathrm{n}+2}-\mathrm{L}^{1}\right)$ is indecomposable.

Proof. Let $G=C * D$. Then by the condition, $\pi_{1}\left(S^{n+2}-\right.$ $\mathrm{K})$ is contained in a conjugate of $C$ or $D[8, \mathrm{p} .245]$. We may suppose that $\pi_{1}\left(S^{n+2}-K\right)$ is contained in $C$. Then the HNN extension of $\mathrm{G}$ with an associated subgroup $<\mu \mid>\cong \mathrm{Z}[8$, p.179]

$$
\mathrm{G}^{*}=\left\langle\mathrm{G}, \mathrm{x}_{\mathrm{m}+1} \mid \mathrm{x}_{\mathrm{m}+1}^{-1} \mu \mathrm{x}_{\mathrm{m}+1}=\mu\right\rangle
$$

is a nontrivial free product $C^{\star} \star D$, where $C^{\star}$ is an HNN extension of $C$

$$
C^{*}=\left\langle C, x_{m+1}\right| x_{m+1}^{-1} \mu x_{m+1}=\mu>
$$

On the other hand, since $\mu=\left[x_{1}, x_{2}, \ldots, x_{m}\right], G^{*}$ is the free product of $\mathrm{H}_{\mathrm{m}+1}$ and $\pi_{1}\left(\mathrm{~S}^{\mathrm{n}+2}-\mathrm{K}\right)$ with an amalgamated subgroup $<\mu \mid>$. Now both $H_{m+1}$ and $\pi_{1}\left(S^{n+2}-K\right)$ are indecomposable, so is $\mathrm{G}^{*}$, and this contradiction completes the proof. 
Remark 1) A satellite $n$-link built from the trivial link 0 and the simple closed curve $l$ representing $\prod_{S \in \hbar_{k}} \alpha_{S^{\prime}}$ where $\Sigma_{k}$ is the family of all the subsets of $I$ consisting of $k$ elements, has the Brunnian property of type $\mathcal{F}_{\mathrm{k}}$.

2) If $n=1$, then the Alexander polynomial of our link $L$ is zero by [12, Theorem 5].

\section{References}

1. H. Brunn, Über Verkettung, Sitzungsberichte der Bayerische Akad. Wiss., Math-Phys. Klasse, 22 (1892), 77-99.

2. H. Debrunner, Links of Brunnian type, Duke Math. J., 28 $(1961), 17-23$.

3. H. Debrunner, Über den Zerfall von Verkettungen, Math. Z., 85 $(1964), 154-168$.

4. R. H. Fox, Some problems in knot theory, Topology of 3-Manifolds and Related Topics, (Prentice-Hall, Englewood Cliffs, 1962), 168-176.

5. J.-C. Hausmann and M. Kervaire, Sur le centre des groupes de noeuds multidimensionnels, C.R.Acad. Sc. Paris, 287 $(1978), 699-702$.

6. J. F. P. Hudson, Piecewise Linear Topology (Benjamin, New York, 1969).

7. T. Kanenobu, Groups of higher dimensional satellite knots, J. Pure Appl. Algebra, 28 (1983), 179-188.

8. R. C. Lyndon and P. E. Schupp, Combinatorial Group Theory (Springer, Berlin-Heidelberg-New York, 1977). 
9. W. Magnus, A. Karrass and D. Solitar, Combinatorial Group Theory (Interscience, New York, 1966).

10. C. D. Papakyriakopoulos, On Dehn's lemma and asphericity of knots, Ann. of Math., 66 (1957), 1-26.

11. D. Rolfsen, Knots and Links (Publish or Perish, Berkeley, CA, 1976 ).

12. G. Torres, On the Alexander polynomials, Ann. of Math., 57 $(1953), 57-89$.

13. T. Yanagawa, Brunnian systems of 2-spheres in 4-space, Osaka J. Math., 1 (1964), 127-132.

Department of Mathematics

Kyushu University 33

Fukuoka, 812

Japan 
30

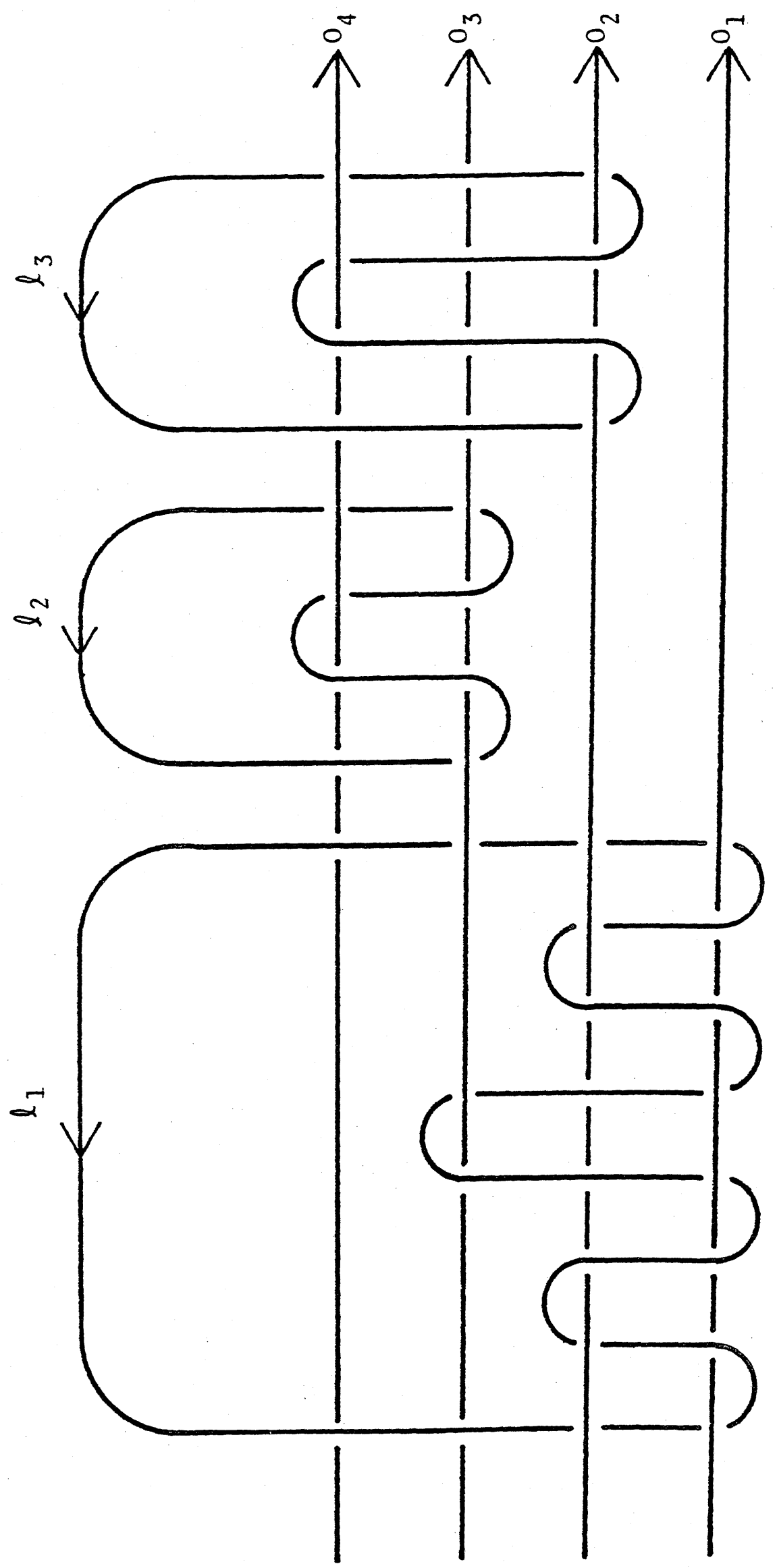

$-10-$ 